\title{
MUS81 Inhibition Increases the Sensitivity to Therapy Effect in Epithelial Ovarian Cancer via Regulating CyclinB Pathway
}

\author{
Ailing Zhong1,*, Hui Zheng1,*, Hongqin Zhang1, Jiajun Sun', Jiabin Shen ${ }^{1}$, Minjie Deng1, Miaomiao Chen ${ }^{1,2}$, \\ Renquan $\mathrm{Lu}^{1,2}$, Lin $\mathrm{Guo}^{1,2}$ \\ 1. Department of Clinical Laboratory, Fudan University, Shanghai Cancer Center, Shanghai, China. \\ 2. Department of Oncology, Shanghai, Medical College, Fudan University, Shanghai, China. \\ * These authors contributed equally. \\ $\triangle$ Corresponding author: Lin Guo and Renquan Lu, Department of Clinical Laboratory, Fudan University, Shanghai Cancer Center, 270 Dongan Road, Xuhui, \\ Shanghai, China. Tel: +86 2164175590 82204; Fax: +86 2164175590 82204; E-mail: guolin500@ hotmail.com and lurenquan@126.com. \\ (c) Ivyspring International Publisher. This is an open access article distributed under the terms of the Creative Commons Attribution (CC BY-NC) license \\ (https://creativecommons.org/licenses/by-nc/4.0/). See http://ivyspring.com/terms for full terms and conditions.
}

Received: 2018.10.20; Accepted: 2019.03.31; Published: 2019.05.21

\begin{abstract}
MUS81 is a key endonuclease involved in homologous recombination (HR) repair after DNA double-strand damage. Structure-specific endonucleases (SSEs) plays a crucial role in DNA replication, repair and transcription, and SSEs are also important for maintaining the secondary structure of DNA; therefore, their activity must be precisely controlled to ensure genome stability. We previously described that MUS81 expression was significantly correlated with CyclinB expression based on protein microarray analysis. CyclinB is a cell-cycle regulatory protein that has been shown to be involved in the activation of DNA damage repair checkpoints by inducing $\mathrm{G} 2 / \mathrm{M}$ phase arrest, promoting apoptosis, and participating in the regulation of chemotherapeutic drug sensitivity by inducing nuclear degradation, as shown by immunofluorescence assays. In this study, MUS81-downregulated cells were generated using lentivirus-mediated RNAi. Our results demonstrated that the inhibition of MUS81 expression activated the CHKI and CyclinB signaling pathways and sensitized ovarian cancer cells to X-ray and Olaparib treatment both in vitro and in vivo. MUS81 may be a potential therapeutic target for epithelial ovarian cancer (EOC).
\end{abstract}

Key words: MUS81, CyclinB, Olaparib, DNA damage repair, EOC

\section{Introduction}

Ovarian cancer is a common gynecological malignancy with high mortality according to cancer statistics from 2018[1]. EOC is the most common pathological type of ovarian cancer, and it has a high recurrence rate. Presently, there is not only a lack of specific diagnostics for ovarian cancer, but also an absence of effective treatment for patients with recurrent ovarian cancer [2]. Although platinum and paclitaxel are the most commonly used chemotherapeutic regimens for the treatment of EOC, their therapeutic effects are limited due to the emergence of drug resistance after the tumor recurs. Fortunately, extensive progress has been made in molecular targeted therapy with the emerging development of novel target-specific drugs as the research on the mechanisms of tumorigenesis and progression grows.

MUS81 is a key molecule that participates in homologous recombination repair. It is a highly conserved gene encoding a structure-specific DNA endonuclease $[3,4]$. As a structure-specific endonuclease, MUS81 is involved in cell cycle regulation and regulates the DNA damage repair process. During S phase of the cell cycle, WEE1 inhibits the association and function of the MUS81-SLX4 complex, and SLX4 cooperates to activate MUS81 during M phase [5]. In the context of DNA double-strand breaks, MUS81 maintains 
genome stability by forming a heterodimer with Eme1/Mms4 and resolving the Holliday junction $(\mathrm{HJ})$ [6]. Maintenance of genomic stability is essential for normal development, cell homeostasis and tumor suppression [7]. Genomic stability relies on the precise execution of DNA replication, chromosome segregation, DNA repair and genomic monitoring mechanisms, and their integration with cell cycle progression and other processes. The regulation of DNA repair by the cell cycle is largely the result of chromatin changes that occur during DNA replication, compression, and dissociation that allow cells to divide properly [8]. CyclinB participates in G2 / $\mathrm{M}$ checkpoint initiation during mitosis [9]; additionally, at the end of mitosis, CyclinB1 is prompted to degrade the complex, allowing cells to complete the replication cycle [10]. The activation of CyclinB is related to the phosphorylation status of CDC25c, CDK1 and other molecules [11]. At the same time, CDK25c and p21 are regulated by CDK25c and p21, which is a downstream molecules of ATM/ATR [12].

Our previous studies confirmed that MUS81 is highly expressed in epithelial ovarian cancer and that its expression was negatively associated with the sensitivity to platinum drugs [13]. It was previously found that the inhibition of MUS81 increased susceptibility to PARP inhibitors through HR deficiency at the cellular level [14]. Using a protein ChIP assay, we identified a significant correlation between MUS81 and CyclinB, which has not been fully elucidated. In this study, we further explored the role of MUS81 and CyclinB in regulating the therapeutic sensitivity of ovarian cancer.

\section{Materials and Methods}

\section{Cell lines}

Human ovarian cancerA2780 and SKOV3 cells were purchased from the Chinese Academy of Sciences Committee (Shanghai, China). Both cell lines were cultured in RPMI-1640 medium supplemented with 10\% fetal bovine serum (FBS) and $1 \%$ penicillin-streptomycin. Cells were incubated at $37^{\circ} \mathrm{C}$ in a humidified atmosphere with $5 \% \mathrm{CO}^{2}$.

\section{Cells treated with $\mathrm{X}$-ray}

Cells were seeded in six-well plates at a density of $3 \times 10^{5}$ cells per well. A2780 and SKOV3 cells were irradiated by X-ray (dose rate: $200 \varepsilon \mathrm{Gy} / \mathrm{min}$, dose: 2 Gy/4 Gy/6 Gy, field: $20 \times 20 \mathrm{~cm}$ ), and a well without $X$-ray irradiation was used as a control. Then, the cells were cultured for 24 hours. Cells were collected and lysed for total protein extraction using IP lysis buffer (Beyotime Biotechnology, Shanghai, China). The expression of MUS81 and CyclinB and other protein molecules were measures by Western blotting, and pH2AX was used as an indicator of double-strand break repair.

\section{Western blot}

Total protein was collected as previously described. Cell lysates were resolved by SDS-PAGE, and proteins were electro transferred to polyvinylidene fluoride membranes (Millipore, USA). The PVDF membranes were blocked with $10 \%$ nonfat milk (Solarbio, Beijing, China). The primary antibodies used included MUS81 (1:200 dilution, Santa Cruz, Texas, USA), $\beta$-actin (1:3000 dilution, Abcam, MA, USA), pH2AX (1:400 dilution, CST, MA, USA), Cyclin B (1:3000 dilution, Abcam, MA, USA), CHK1 (1:200 dilution, Santa Cruz, Texas, USA), CDC25C (1:200 dilution, Santa Cruz, Texas, USA ), pCHK1(1:1000 dilution, CST, MA, USA), pCDC25C(1:1000 dilution, CST, MA, USA), RAD51(1:3000 dilution, Abcam, MA, USA), BCL2(1:3000 dilution, Abcam, MA, USA), and BAX (1:3000 dilution, Abcam, MA, USA).

Table 1. Sequences of primers and targets

\begin{tabular}{ll}
\hline Primers/targets & Sequences \\
\hline MUS81 qRT-PCRforward & 5'-CTGAAGCGCTGTGGTCTG-3' \\
MUS81 qRT-PCR reverse & 5'-AGTGTTGGTGACAGCCTG-3' \\
$\beta$-actin qRT-PCR forward & 5'-AAGGTGACAGCAGTCGGTT-3' \\
$\beta$-actin qRT-PCR reverse & 5'-TGTGTGGACTTGGGAGAGG-3' \\
CyclinB qRT-PCR forward & 5'-TCTGGATAATGGTGAATGGACA-3' \\
CyclinB qRT-PCR reverse & 5'-CGATGTGGCATACTTGTTCTTG-3' \\
Cdc25C qRT-PCR forward & 5'-GAAGCATCTGAGCAGTCCCATTAC-3' \\
Cdc25C qRT-PCR reverse & 5'-CTGGCACCGTTGGCAGCACAC-3' \\
shMUS81-1 & 5'-ACACTGCTGAGCACCATTAAG-3' \\
shMUS81-2 & 5'-GCAGCCCTGGTGGATCGATAC-3' \\
shCtrl (scrambled sequence) & 5'-CCTAAGGTTAAGTCGCCCTCG-3' \\
\hline
\end{tabular}

\section{Establishment of MUS8 1 downregulated cell lines}

A MUS81 lentivirus expressing a specific RNAi interference sequence was first designed. The nucleotide sequences were cloned into the AgeI and EcoRI sites of a pLKO.1-puro vector (Addgene, MA, USA) to generate the pLKO.1-puro-MUS81 (shMUS81-1 and shMUS81-2) and pLKO.1-purocontrol (shCtrl) (Table 1) recombinant vectors. Lentiviruses were packaged by transfecting $293 \mathrm{~T}$ cells with the described lentivirus recombinant vectors, the packaging plasmid psPAX2, and the envelope vector pMD2.G (Addgene, MA, USA) using Lipofectamine 2000 transfection reagent (Invitrogen, CA, USA). Culture medium containing lentiviral particles was collected after 48 hours. Ovarian cancer cells were transfected with $1 \times 10^{6} \mathrm{IFU} / \mathrm{ml}$ lentivirus in $8 \mathrm{mg} / \mathrm{ml}$ Polybrene (Sigma-Aldrich, MO, USA) for 24 hours. Stably transfected cells were selected using $1 \mathrm{mg} / \mathrm{ml}$ puromycin (Sigma-Aldrich, MO, USA) for 7 days, and 
the RNAi knockdown efficiency was verified by Western blot.

\section{Quantitative RT-PCR}

Preparation of the MUS81 down-regulated cell lines. RNA was isolated from cells using TRIzol ${ }^{\circledR}$ reagent (Thermo Fisher Scientific, Shanghai, China) and reverse transcribed using a PrimeScript ${ }^{\circledR}$ RT reagent Kit (TaKaRa, Shiga, Japan) according to the manufacturer's protocol. RT-PCR was performed in a $10 \mu \mathrm{l}$ reaction solution containing $10 \mathrm{ng}$ cDNA, 0.1 $\mathrm{mmol} / \mathrm{l}$ primer (Table 1), and $5 \mathrm{ml}$ 2xSYBR Premix Ex Taq (TaKaRa, Shiga, Japan). PCR amplification was performed at $95^{\circ} \mathrm{C}$ for 5 minutes, followed by 40 cycles at $95^{\circ} \mathrm{C}$ for 15 seconds, and $65^{\circ} \mathrm{C}$ for $40 \mathrm{sec}$, using a Mastercycler ${ }^{\circledR}$ ep realplex (Quantstudio $\mathrm{dx}$, Thermo Fisher Scientific). The MUS81, CyclinB and Cdc25C transcript abundances were expressed relative to $\beta$-actin as a control.

\section{Cell cycle and apoptosis assay}

Cells were seeded onto six-well plates at a density of $4 \times 10^{5}$ cells per well and were then irradiated with X-ray (dose rate: $200 \varepsilon \mathrm{Gy} / \mathrm{min}$, dose: 4 Gy, Field: $20 \times 20 \mathrm{~cm}$ ). To examine apoptosis, $1 \times 10^{5}$ cells were collected and washed twice with $4^{\circ} \mathrm{C}$ PBS. Then, the cells were resuspended in $1 \times$ Annexin $\mathrm{V}$ binding buffer and stained with propidium iodide (PI) and Annexin V according to the instructions of the Annexin V-fluorescence apoptosis detection kit I (BD Biosciences PharMingen, USA). For cell cycle analysis, transduced cells were harvested, fixed in $70 \%$ alcohol overnight at $4^{\circ} \mathrm{C}$ and then incubated with $500 \mathrm{ml}$ of PI (BD Pharmingen ${ }^{\mathrm{TM}}$, USA) for 15 minutes in the dark. Finally, apoptosis and the cell cycle were analyzed by flow cytometry (BD, Caliburn), and the assays were repeated three times.

\section{In vivo experiments}

BALB/c mice that were 5-8 weeks old were selected, and their body weight was approximately 18-20 g. The in vivo experiments with shCtrl and shMUS81-1 A2780 cells were performed as previously described. On day 0, ovarian cancer cells were injected subcutaneously into the back or flank, respectively, of the mice. The tumor size and weight of the mice were measured daily. When the calculated tumor volume reached approximately $1.0-1.2 \mathrm{~cm}^{3}$, mice were grouped and subjected to the first treatment. Experimental groups (5 mice/group) were administered Olaparib (50 $\mathrm{mg} / \mathrm{kg}$ ) daily for one week. Control mice (5 mice/group) were treated in the same way as the experimental mice, but were not given Olaparib. Tumor tissue was recovered on day 7 after the completion of Olaparib administration (three weeks after the start of the experiment). The expression of MUS81 and CyclinB were determined by measuring protein levels in the tumor tissue samples.

\section{Immunohistochemistry}

Immunohistochemistry (IHC) was performed using an anti-MUS81 mouse monoclonal antibody, diluted with PBS at 1:50 (Abcam, MA, USA). In brief, each section (4- $\mu \mathrm{m}$-thick) was dewaxed and hydrated, followed by inhibition of endogenous peroxidase activities with methanol containing $0.3 \% \mathrm{H}_{2} \mathrm{O}_{2}$. After antigen retrieval and cooling down, the sections were blocked with $1 \%$ BSA and incubated overnight at $4{ }^{\circ} \mathrm{C}$ with primary antibody. On the second day, the sections were incubated with HRP-conjugated secondary antibody (Shanghai Long Island Biotech, Shanghai, China) for $1 \mathrm{~h}$ at room temperature, followed by treatment with diaminobenzidine and counterstaining with hematoxylin.

\section{Immunofluorescence}

For immunofluorescence studies, $5 \times 10^{5}$ cells were seeded in 24-well plates on glass coverslips. Olaparib $(5 \mu \mathrm{mol} / \mathrm{l})$ was added to cells, and a well without Olaparib addition was used as a control. The cells were cultured for 48 hours. After the cells adhered, they were washed with PBS and fixed with $4 \%$ formaldehyde at room temperature. The slides with the fixed cell slides were washed with PBS, and then methanol and acetone were added at a 1:1 ratio, which was followed by blocking with $1 \%$ BSA. The cells were then incubated with antibodies against MUS81 (1:200 dilution, Santa Cruz, Texas, USA) and CyclinB (1:200 dilution, Abcam, MA, USA) overnight at $4^{\circ} \mathrm{C}$. After washing, the cells were labeled with Alexa Fluor 488-conjugated secondary antibody (Invitrogen, CA, USA) and examined under a fluorescence microscope (Leica, China, SP5).

\section{Statistical analysis}

Data are presented as the mean \pm SD of three independent experiments, and SPSS 20.0 software was used for statistical analyses. The differences between groups were examined by analysis of variance (ANOVA) and Student's t-tests. $P<0.05$ was considered to indicate statistical significance.

\section{Results}

\section{MUS81 and CyclinB are involved in DNA double-strand break repair in epithelial ovarian cancer.}

MUS81 is a highly expressed gene in epithelial ovarian cancer, and its overexpression is associated with poor prognosis and high levels of resistance to Olaparib, as shown by Oncomine database analysis 
(Figure 1A). MUS81 is a key endonuclease involved in the homologous recombination repair of DSBs. In this study, we established a double-strand break injury model by $\mathrm{X}$-ray irradiation and employed $\mathrm{pH} 2 \mathrm{AX}$ as a marker of double-strand break repair. We showed that the protein expression of MUS81 and CyclinB gradually increased with increasing irradiation. During this process, the CHK1 signaling pathway was not activated, the expression of the downstream molecule Cdc25C increased and the phosphorylation of Cdc25C (Ser-216) was inhibited(Figure 1B). The results showed that both MUS81 and CyclinB molecules participated in the HR repair pathway and regulated the activation of the CHK1 signaling pathway of CyclinB.

\section{Downregulation of MUS81 increases the sensitivity of epithelial ovarian cancer cells to radiotherapy.}

MUS81 downregulation in A2780 and SKOV3 cells were performed using a lentivirus-mediated method. The expression of CyclinB at the G2/M phase checkpoint was detected by Western blotting, and CHK1 (Ser-345) and Cdc25C (Ser-216) were not activated (Figure 2A). Consistent results were observed at the RNA level by RT-PCR (Figure 2B). Flow cytometry showed that G1 phase arrest was observed after MUS81 downregulation (Figure 2C). This result indicated that G1 phase arrested occurred after downregulation of MUS81, and the G2/M phase checkpoint protein CyclinB was not activated, which was consistent with changes in protein levels. Further, we performed X-ray irradiation on MUS81-downregulated cell lines and found that inhibition of MUS81 expression increased the sensitivity of epithelial ovarian cancer cells to X-ray. Flow cytometry revealed that apoptosis increased and the cell cycle arrested at G2/M phase (Figure 3A,B). At the protein level, we observed an increase in the phosphorylation of CHK1 (Ser345) and Cdc25C (Ser216), activation of the G2/M phase checkpoint, and inhibition of CyclinB expression (Figure 3C).
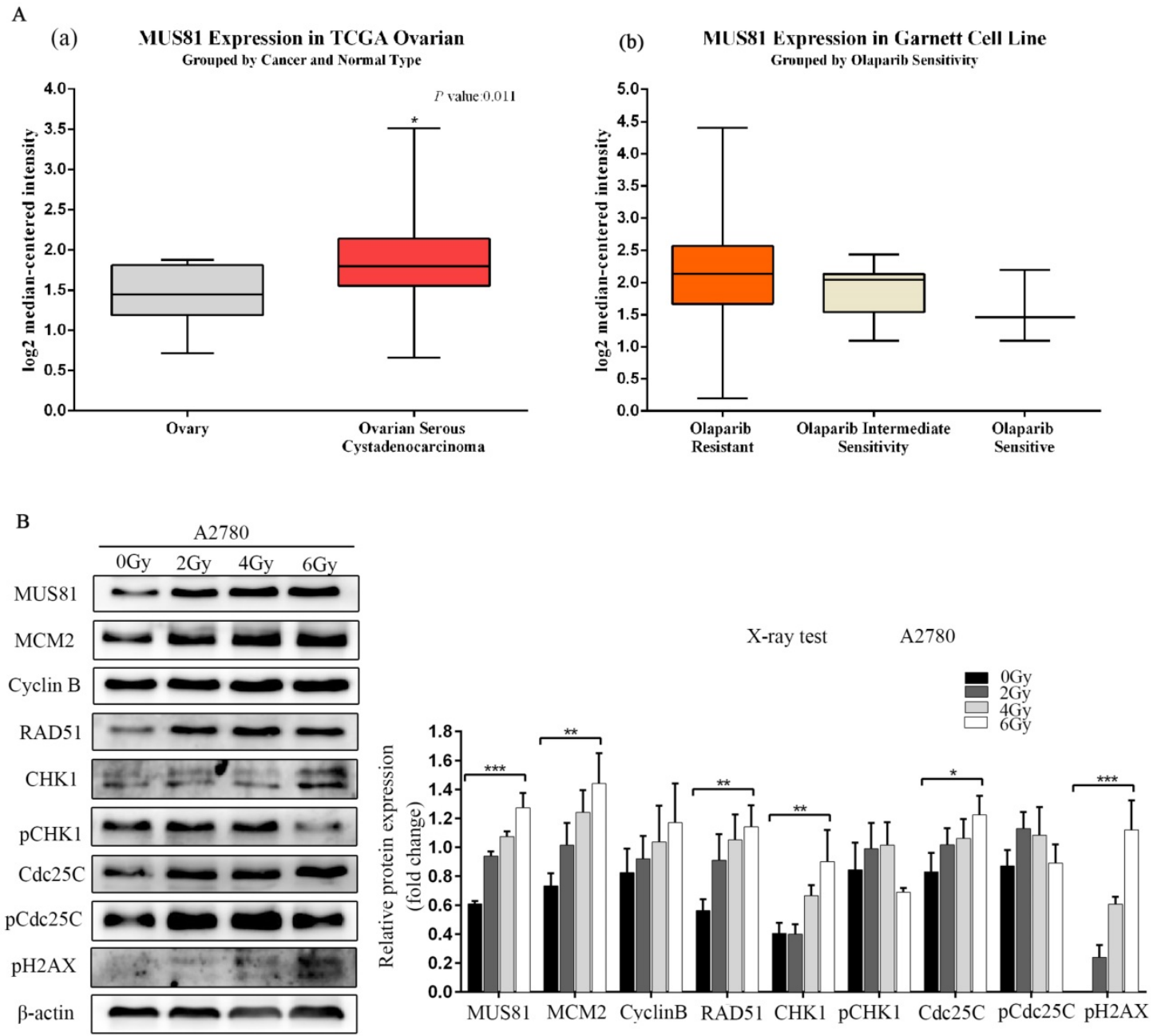

Figure 1. Overexpression of MUS81 in epithelial ovarian cancer (EOC) and the association with Olaparib sensitivity. (A) Oncomine data analysis for MUS81 in ovarian cancer. (a) mRNA expression of MUS81 was overexpressed in ovarian cancer compared to normal ovarian tissue. The data were retrieved from the TCGA database. (b) MUS81 was overexpressed in Olaparib-resistant tissues compared to the expression of other groups. The data were retrieved from the Garnett Cell Line database. (B) Both MUS81 and CyclinB molecules participated in the HR repair pathway. $* P<0.05$. Data are presented as the mean \pm SD of three independent experiments. 
Phosphorylation of $\mathrm{CHK} 1$ promotes the increase in pCdc25C (Ser216), prevents Cdc25C arrest in G2/M phase and cell apoptosis increase the dephosphorylation of $\mathrm{Cdc} 2$, and inhibits the sensitivity of MUS81-deficient ovarian cancer to formation of CyclinB and CDK1 complexes. Cell cycle

radiotherapy.

A

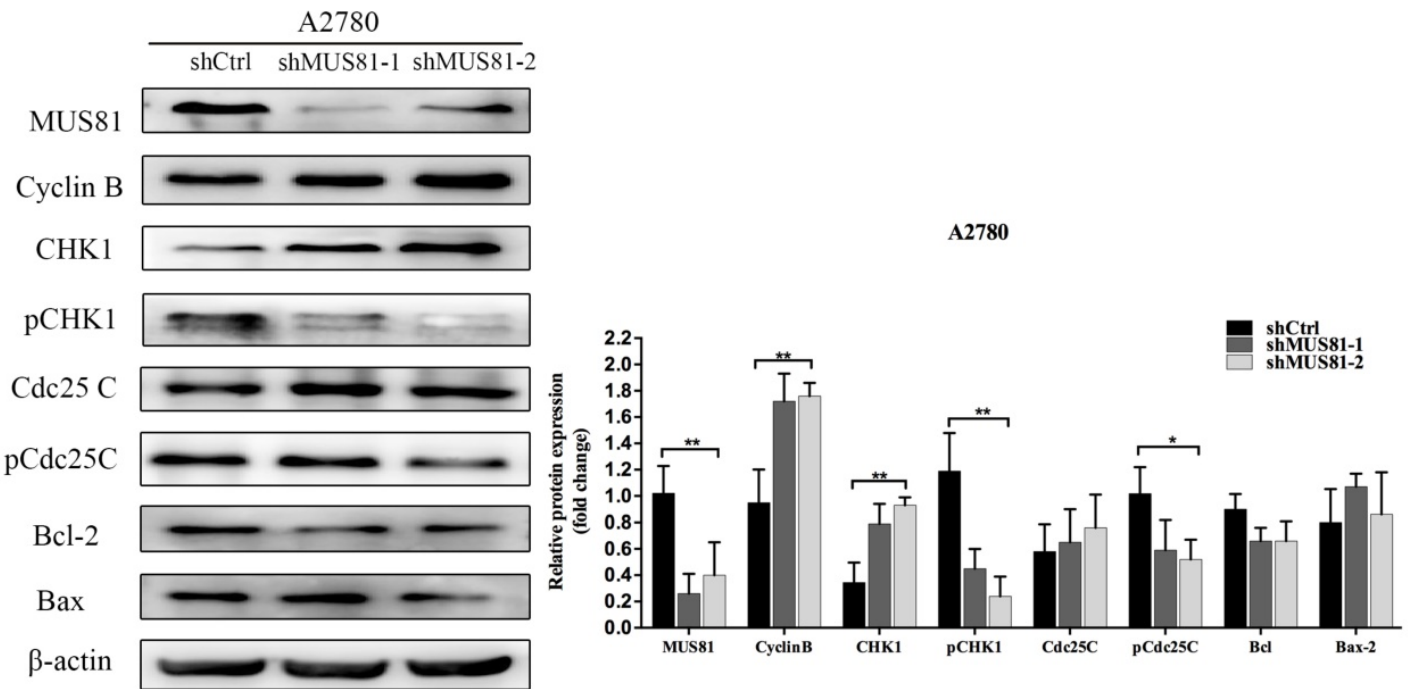

B
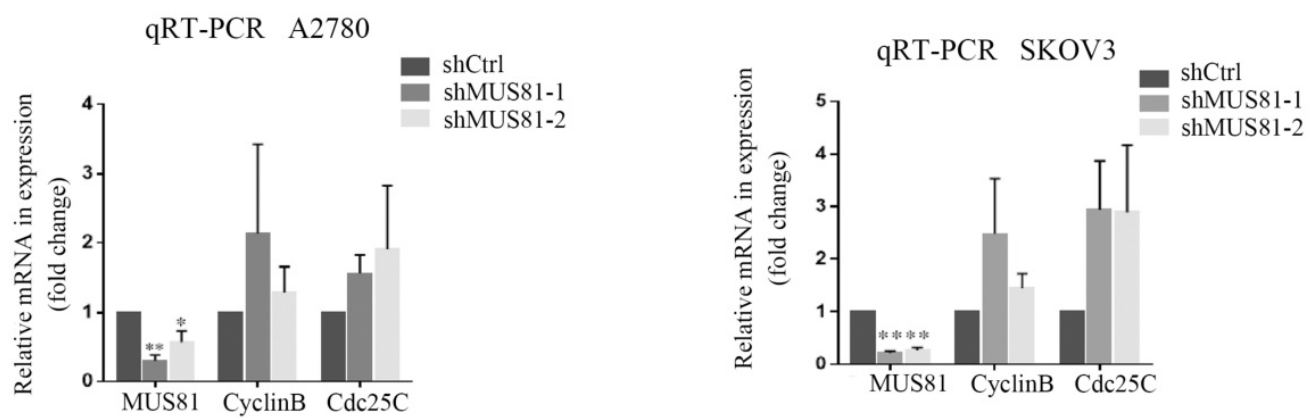

C

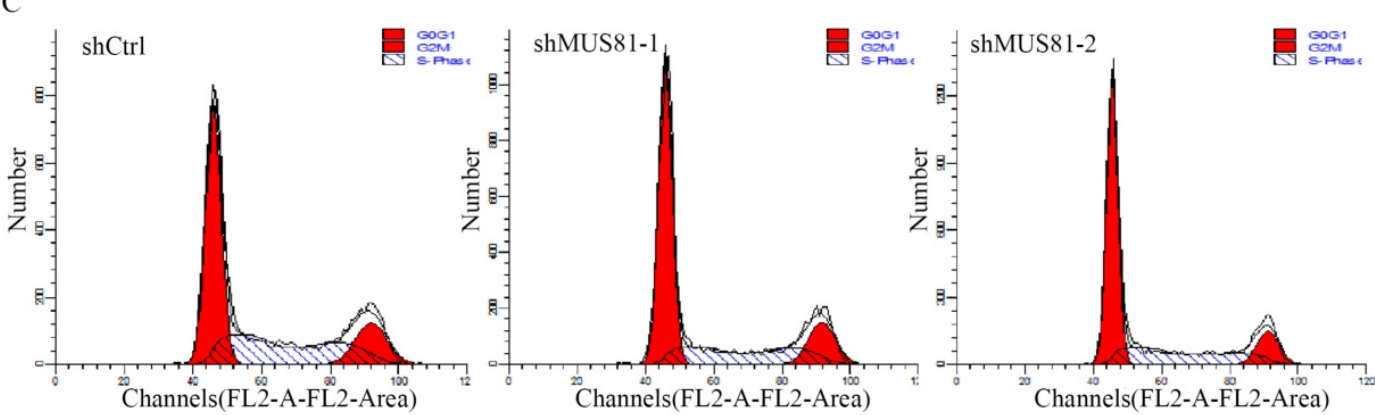

Cell cycle A2780

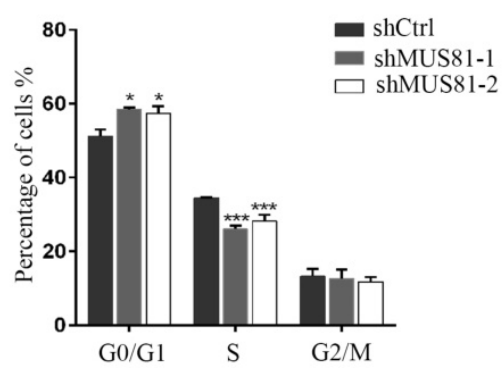

Figure 2. MUS81 regulates CyclinB expression and the cell cycle. (A, B) Lentivirus-mediated RNAi constructs were used to generate the MUS81-downregulated cell lines, and increased expression of CyclinB was revealed by Western blotting and GRT-PCR. (C) The effect of MUS81-downregulation on cell cycle distribution. The cell cycle was analyzed by flow cytometry, and the data are presented as the mean \pm SD of three independent experiments. $(* P<0.05, * * P<0.01$, $* * * P<0.001)$. 


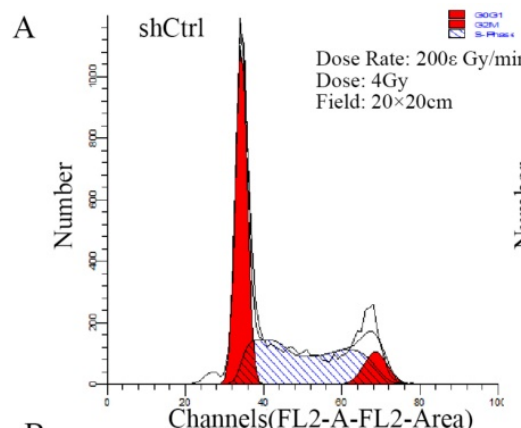

B Dose Rate: $200 \varepsilon \mathrm{Gy} / \mathrm{min}$
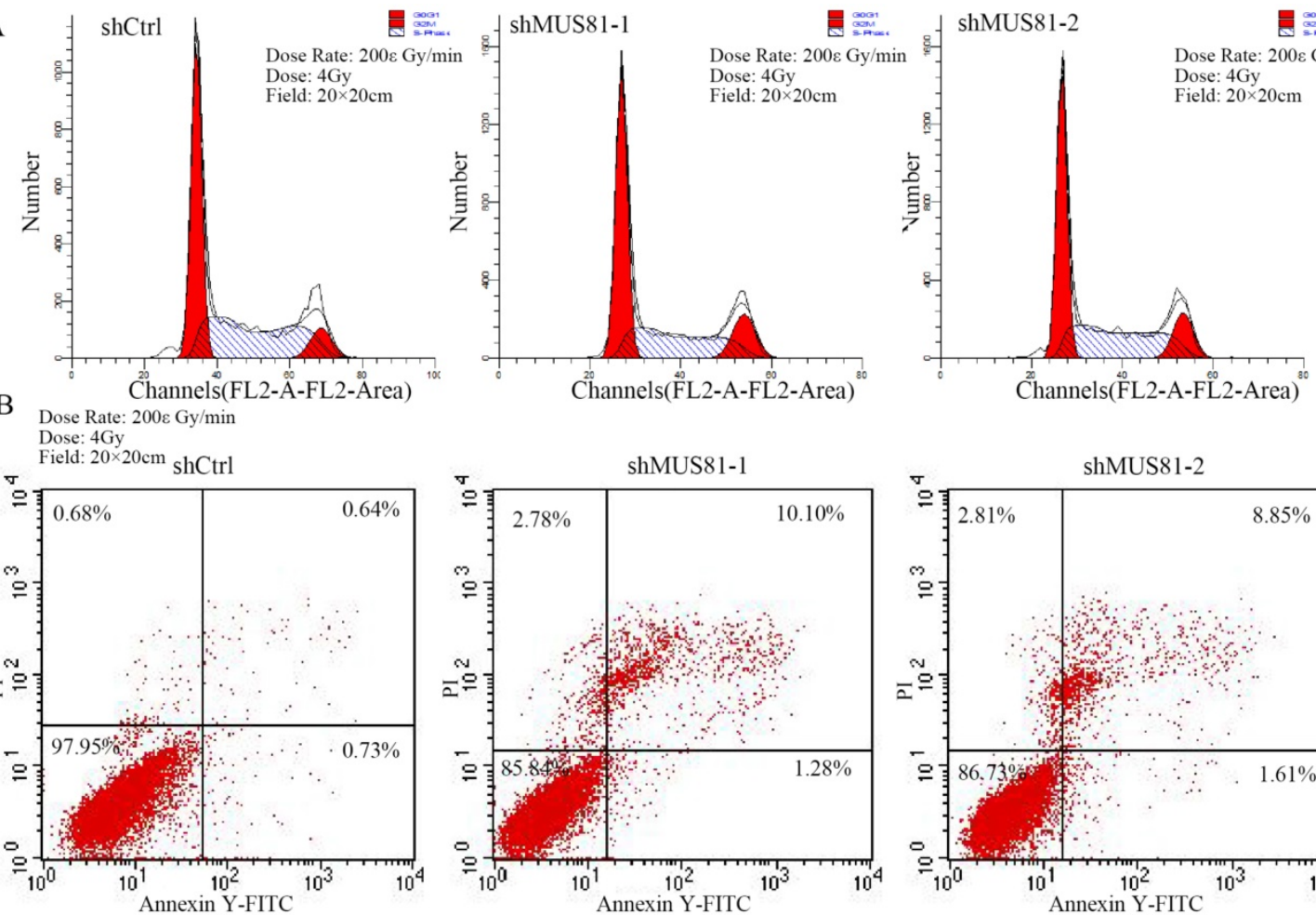

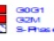

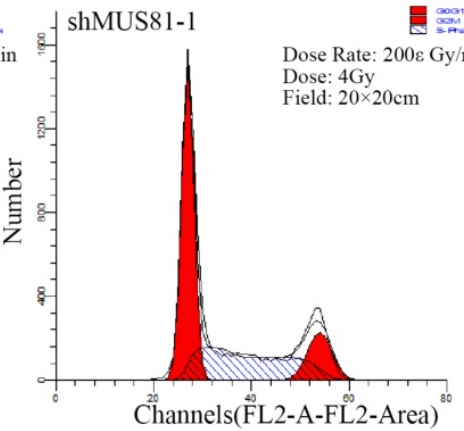

ose Rate: $200 \varepsilon \mathrm{Gy} / \mathrm{min}$ Dose: $4 \mathrm{~Gy}$

$20 \times 20 \mathrm{~cm}$

Cell cycle A2780
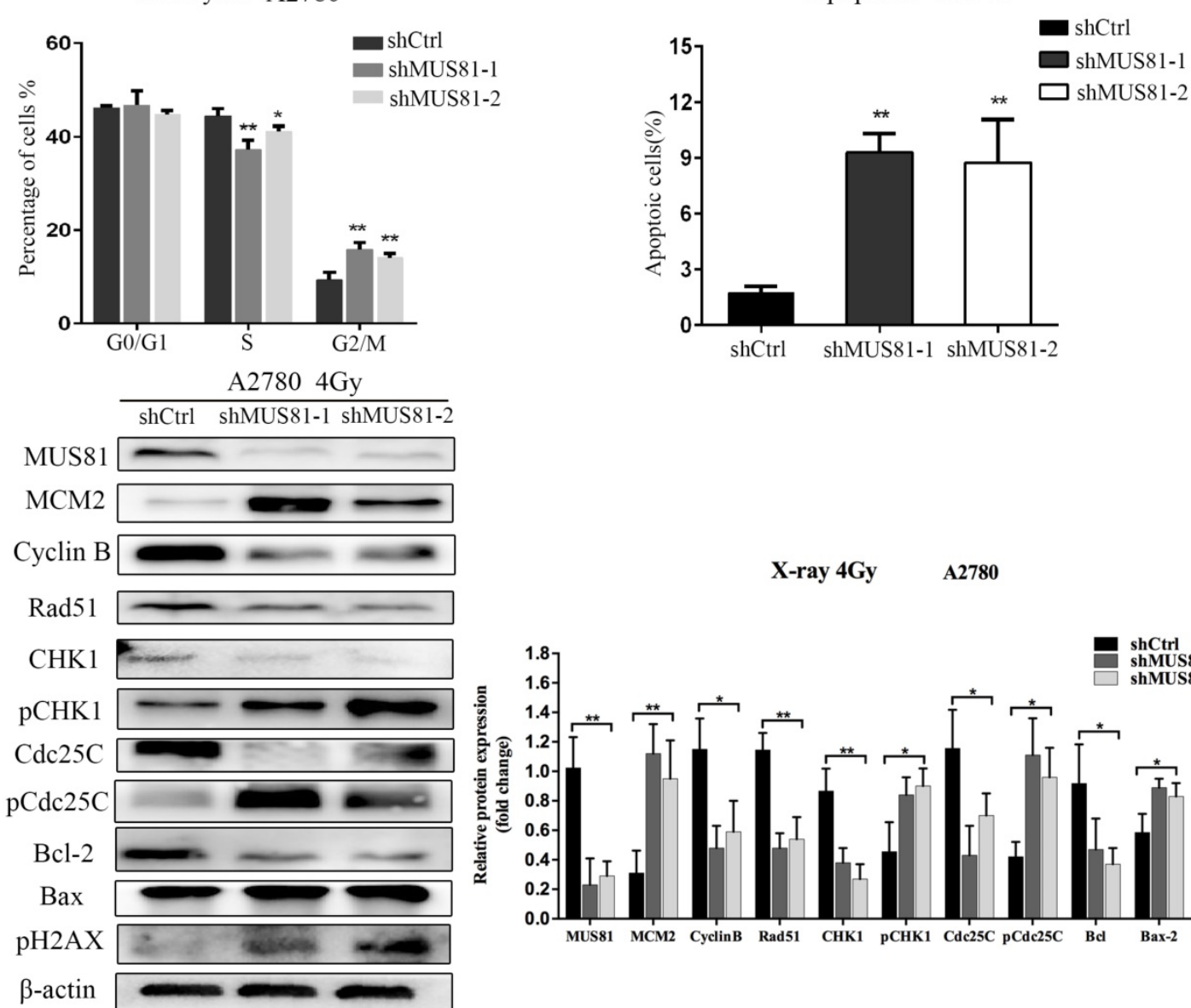

X-ray 4Gy $\quad$ A2780

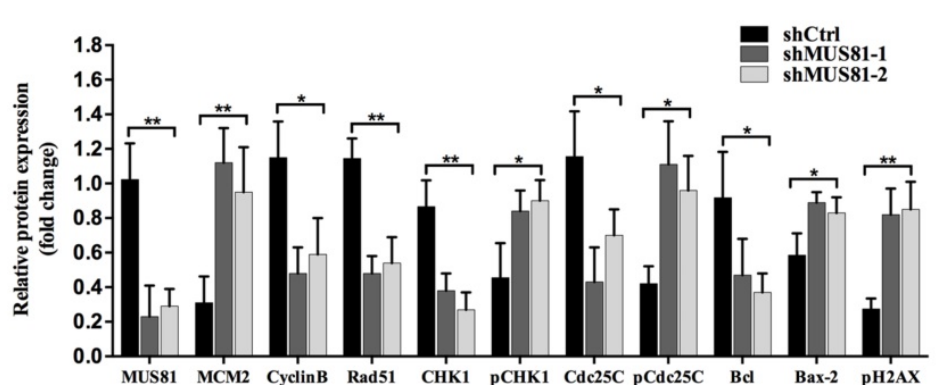

Figure 3. Downregulation of MUS81 increases the sensitivity to X-ray by regulating the CyclinB pathway. (A, B) The effect of X-ray on cell cycle distribution. shCtrl and shMUS8I cells were irradiated with 4 Gy X-ray. Cell cycle and cell apoptosis were analyzed by flow cytometry, and the data are presented as the mean \pm SD of three independent experiments. (C) The effect of X-ray on protein expression. Western blot analysis of MUS81 and CyclinB pathway member expression levels in A2780 cells after treatment with $4 \mathrm{~Gy} X$-ray compared to the expression levels of a blank control. $\beta$-actin was used as the loading control. The bars (fold change) represent the relative expression of the target protein relative to $\beta$-actin. 
A

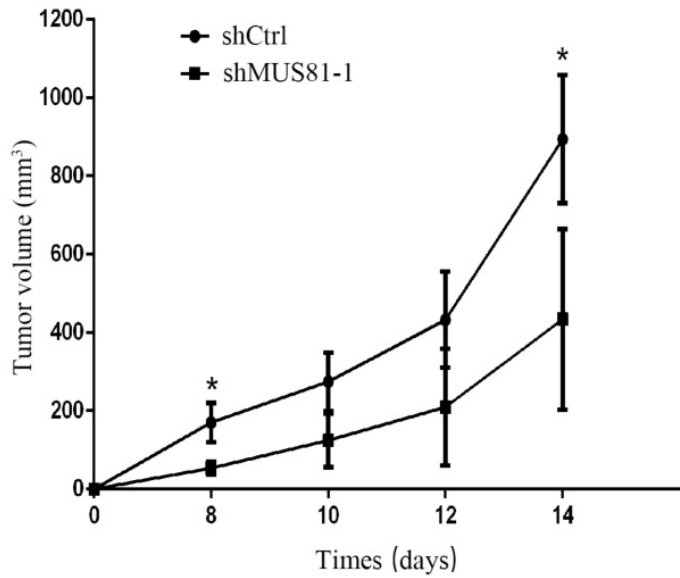

B

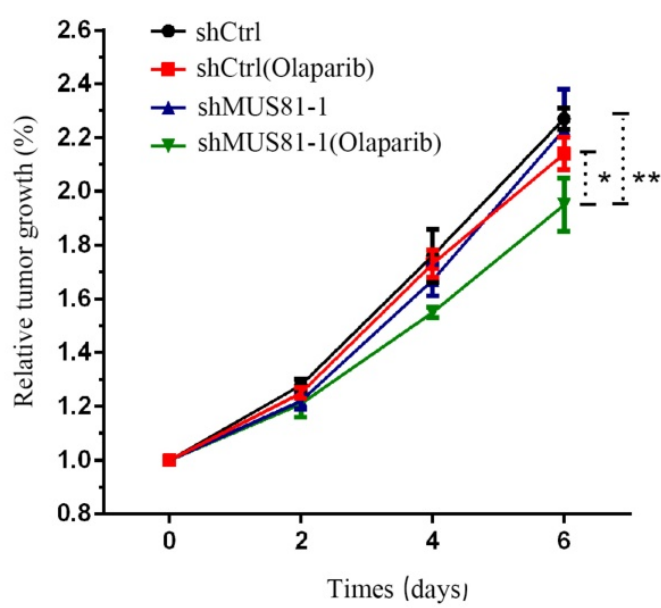

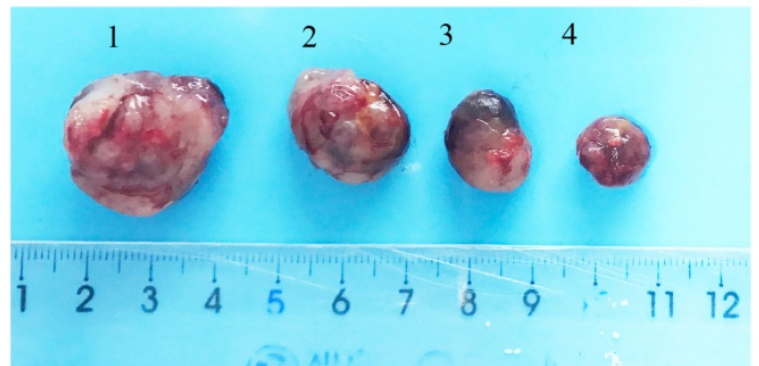

1.shCtrl Olaparib-

2.shMUS81-1 Olaparib-

3.shCtrl Olaparib+

4.shMUS81-1 Olaparib+

Figure 4. Downregulation of MUS81 increases drug sensitivity to Olaparib. (A) Knockdown of MUS81 inhibited tumor growth in vivo. Injection of MUS81 downregulated A2780 cell lines. Xenografted tumor volume was measured every two days. $* P<0.05$, shCtrl vs shMUS81-1.(B) The relative size of the tumor after administration. Representative images of xenografted tumors in the four groups.

\section{In vivo experiments confirm the sensitization of MUS8 1-downregulated ovarian cancer to Olaparib through CyclinB regulation.}

Previous studies demonstrated that inhibition of MUS81 can reduce HR activity and that MUS81-/ HREOC cells are more sensitive to PARP inhibitors than wild type ovarian cancer cells in vitro. In the present study, we investigated the role of MUS81 in vivo in BALB/c nude mice by subcutaneously injecting shCtrl and shMUS81-1 cells into mice in the construct control and MUS81-deficient ovarian cancer groups, respectively. Our results showed that the tumor growth rate of the MUS81-deficient mice was significantly lower than that of the control group (Figure 4A). Olaparib was injected intraperitoneally at a dose of $50 \mathrm{mg} / \mathrm{kg}$ after the tumor had reached an appropriate size $\left(1.0-1.2 \mathrm{~cm}^{3}\right)$. One week after treatment, the tumor size of the Olaparib drug-resistant MUS81 deficient group was significantly higher than that of the control group. No tumor growth was observed in the chemotherapy-treated group (Figure 4B). Next, we sought to investigate the role of MUS81 in Olaparib sensitivity and its relationship to activation of the CyclinB signaling pathway. The protein expression of
MUS81 and CyclinB was detected by immunohistochemistry, and a comparison with the control group was performed. Elevated CyclinB expression was observed in the murine MUS81-downregulation group, and MUS81 was expressed in the cytoplasm. The MUS81downregulation group was more sensitive to Olaparib and the CyclinB protein expression was significantly lower than that of the control group (Figure 5). In this study, in vivo experiments confirmed that inhibition of MUS81 can increase the sensitivity of epithelial ovarian cancer to Olaparib. In BRCA wild-type ovarian cancer, HR defects are produced by targeting the inhibition of MUS81, and PARP inhibitors are used to avoid platinum drug resistance.

\section{CyclinB regulates sensitivity to radiotherapy and chemotherapy through nuclear entry.}

We have found that mus 81 regulated the chemotherapy sensitivity to Olaparib through the activation of the Cyclinb pathway in vivo, and we further validated the regulatory mechanisms of MUS81 in vitro. According to Western blot analysis, 5 $\mu \mathrm{mol} / 1$ Olaparib inhibited MUS81 expression in shCtrl and shMUS81 ovarian cancer cells. The 
expression of CyclinB in shMUS81 cells was significantly reduced compared with that of shCtrl cells (Figure 6A). The action of Olaparib promoted the phosphorylation of CHK1 and Cdc25C, and G2/M phase checkpoint regulated the reduced expression of CyclinB. The expression of CyclinB in shCtrl and shMUS81-1 cells after Olaparib treatment was also detected by cell immunofluorescence. We observed that the expression of CyclinB was inhibited by activation of the CyclinB pathway in shMUS81-1 cells and that CyclinB entered the cell nucleus from the cytoplasm (Figure 6B). The activity of nuclear CyclinB is relatively weak in comparison to the activity of the cytoplasmic protein. These results suggest that CyclinB participates in the process of DNA damage repair by nuclear translocation.
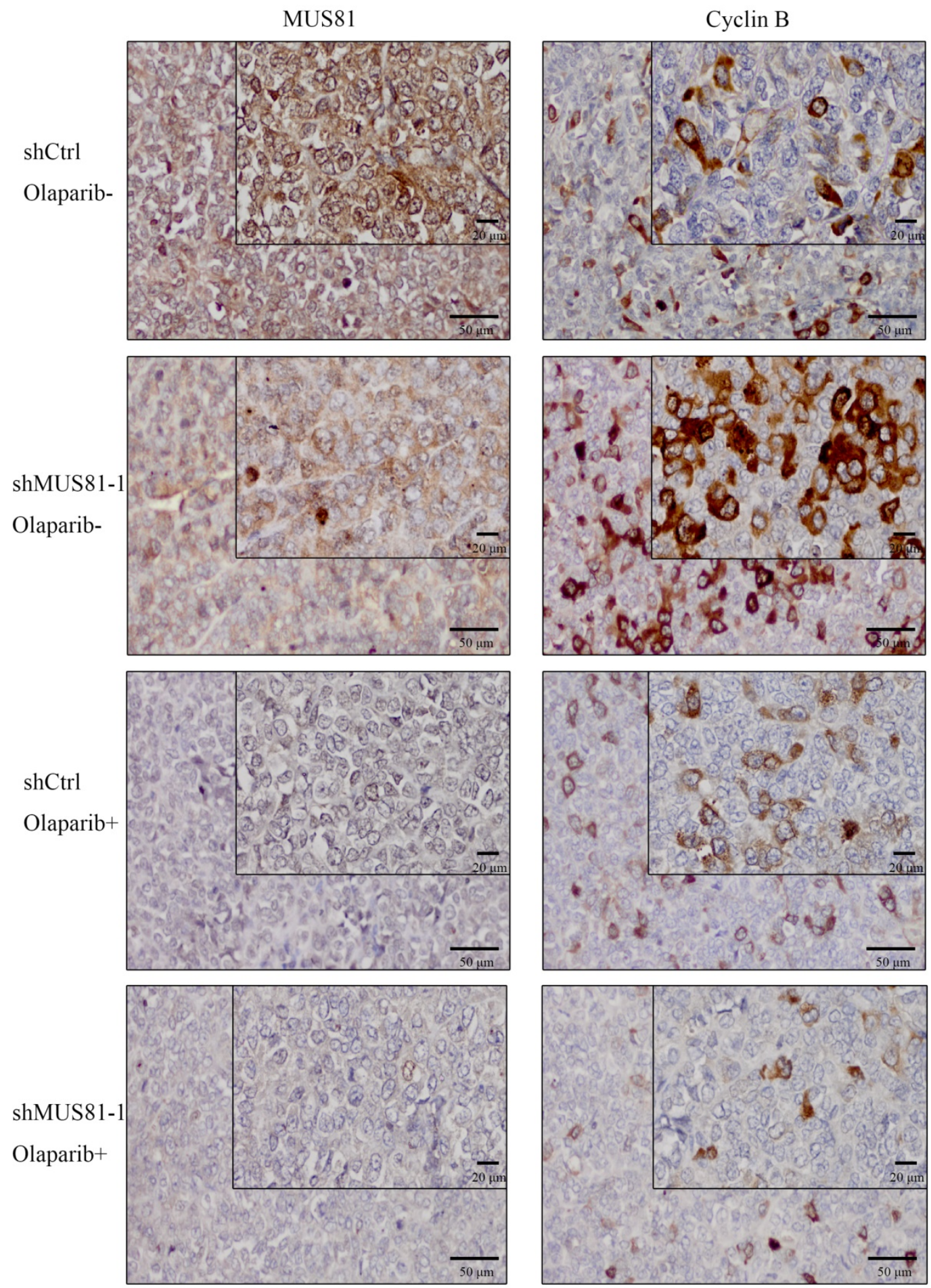

Figure 5. Downregulation of MUS81 increases drug sensitivity to Olaparib through CyclinB regulation. Immunostaining of the MUS8land CyclinB proteins in tumor tissues. In the control group, the expression of CyclinB increased after downregulation of MUS81. The expression of CyclinB was suppressed in the treatment group compared to that of the control group. 
Based on the above findings, we concluded that inhibition of MUS81 could improve the sensitivity of epithelial ovarian cancer to radiotherapy and Olaparib treatment and may be associated with the CyclinB signaling pathway. Activation of phosphorylation of $\mathrm{CHK} 1$ and $\mathrm{Cdc} 25 \mathrm{C}$ leads to CyclinB degradation and thus inhibited the formation of CyclinB and CDK1 complexes, arresting the cell cycle at G2/M phase and promoting cellular apoptosis (Figure 7).
A

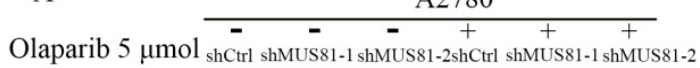

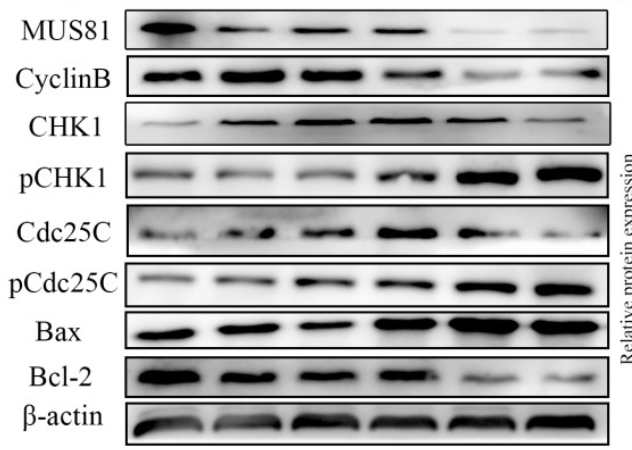

$\mathrm{B}$
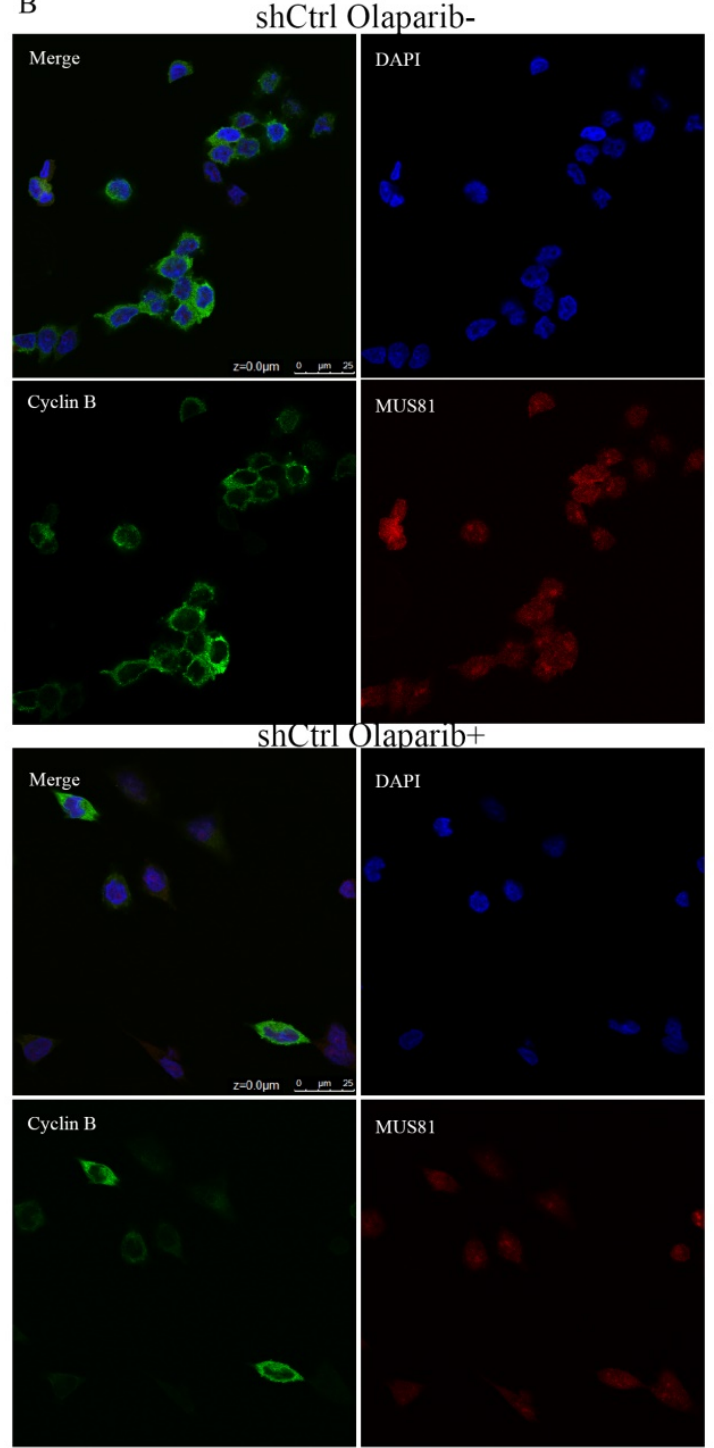

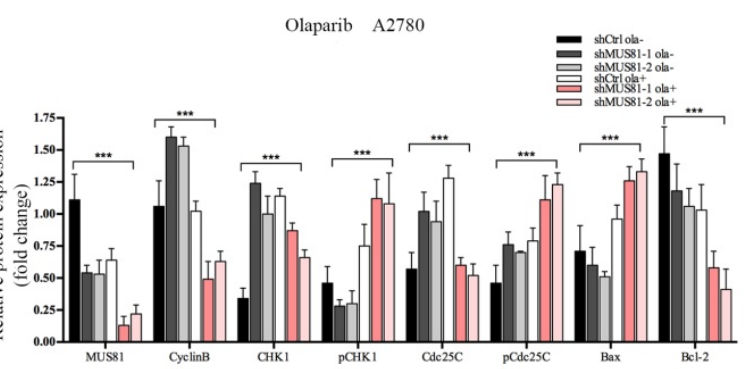

shMUS81-1 Olaparib-

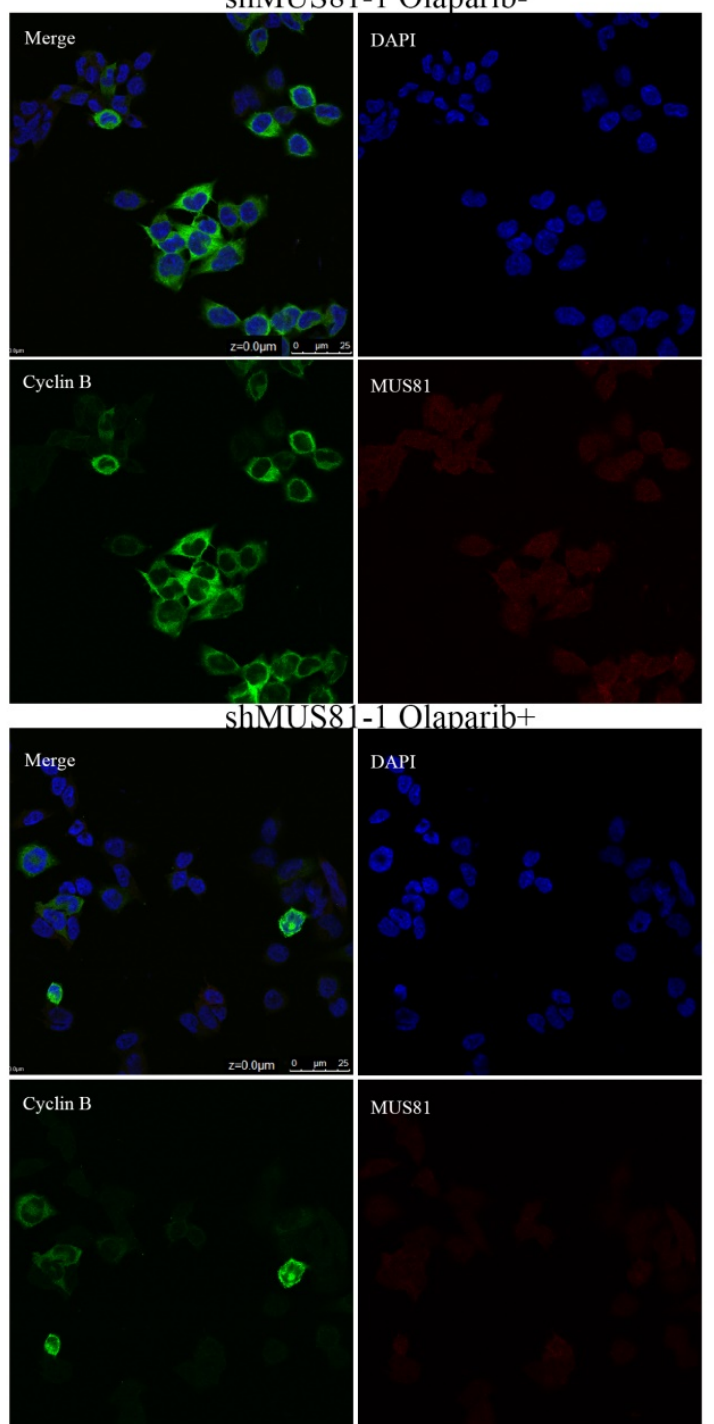

Figure 6. The effect of Olaparib on protein expression. (A) Western blot analysis of MUS81 and CyclinB pathway member expression levels in A2780 cells after treatment with $5 \mu \mathrm{mol} / / \mathrm{O}$ Olaparib compared to that of a blank control. $\beta$-actin was used as the loading control. The bars (fold change) represent the relative expression of the target protein relative to $\beta$-actin. (B) Confocal microscopy revealed the localization of the MUS81 and CyclinB proteins in the shCtrl and shMUS81-1 cells of A2780 cell lines. 


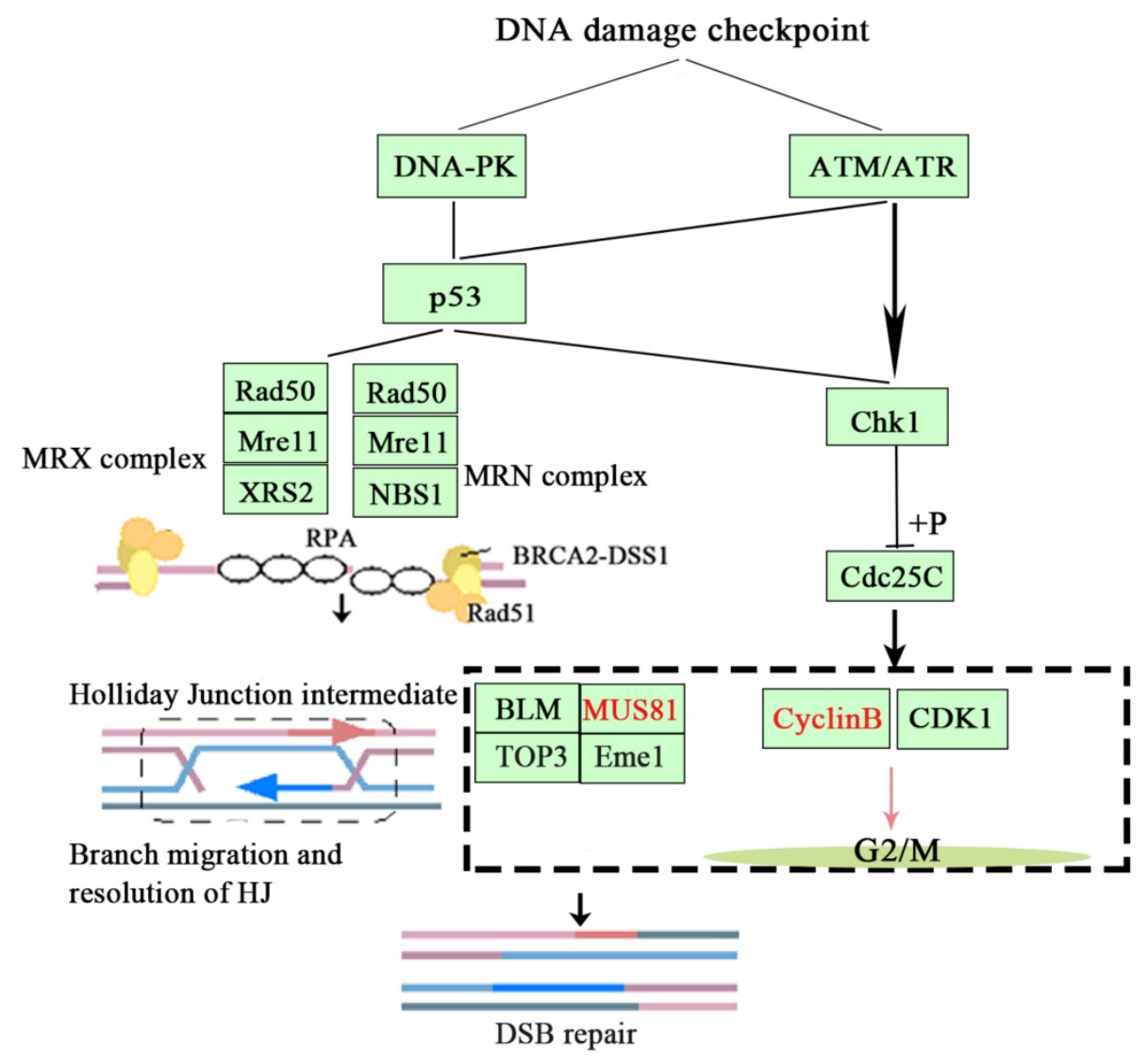

Figure 7.The signaling mechanism of MUS81 and CyclinB.

\section{Discussion}

Structure-specific endonucleases (SSEs), are controlled as a part of genome maintenance pathways in eukaryotes, utilizing elaborate mechanisms that regulate the members of the major SSE families, including the XPF family and MUS81-dependent nucleases, and the FEN1, XPG and XPG-like endonuclease 1 (GEN1) enzymes, during processes such as DNA adduct repair, Holliday junction processing and replication stress [15]. Homologous recombination repair is strictly regulated by the cell cycle; MUS81 specifically cleaves replication forks at the HJ, the SLX1-SLX4-MUS81-EME1-XPF-ERCC1 complex (SMX complex) mediates HJ lysis, and MUS81 activity affects sister chromatids. After the separation of the later formed homologous recombination ultrafine bridges (HR-UFBs) that form later, the breakage of cell bridges during cell division leads to the activation of DNA damage checkpoints [16]. It is critically important that after DNA damage is sustained, the cell division of the daughter cells is arrest to allow for sufficient repairs, and to prevent the production of mutant cells that propagate incorrect genetic information. The regulation of homologous recombination repair mechanisms is influenced by cell cycle-regulated proteins. Our previous study found that downregulation of MUS81 significantly inhibited the activity of HR and caused S phase arrest in the proliferation cycle of ovarian cancer cells. When HR-deficient ovarian cancer cells were treated with X-ray irradiation or Olaparib, G2/M cell cycle phase arrest occurred, and initiation of apoptosis increased the sensitivity of tumor cells to radiotherapy and chemotherapy. CyclinB operates at the G2/M transition and during M-phase progression. Culligan et al. proposed that after gamma irradiation CyclinB was as strongly induced as Rad51 after gamma irradiation, which is an essential gene for DNA double-strand break repair by HR [17]. The transcriptional induction of it was found to depend on the Ataxia Telangiectasia Mutated (ATM) and ATM- and Rad3-related (ATR) kinases that play a central role in sensing and triggering DNA damage responses. Consistent with a potential role in the DNA damage response, CyclinB1 was found to be activated in several mutants that suffered from DNA double-strand breaks [18]. In HR-regulated mitosis, HR substrates may require the phosphorylation of different CDK1-CyclinB1 complexes. The initiation of the DNA damage checkpoint requires the CHK1/2 regulation. We verified by Western blot that MUS81-downregulation ovarian cancer cells initiated cell cycle damage repair detection via CHK1. A 
downstream molecule of CHK1 is CDC25C, and it was found that CHK1 and CDC25C participated in the activation and regulation of signaling pathways via phosphorylation.

Previous studies have suggested that the inhibition of MUS81 activity could affect the sensitivity of colon cancer to chemotherapeutic drugs through activation of the CHK1 pathway [19]. Human CHK1 is a recently identified homologue of the Schizosaccharomyces pombe checkpoint kinase gene, which is required for G2 arrest in response to DNA damage [20, 21]. CHK1 phosphorylates the dual-specificity phosphatase CDC25C at Ser-216, and this may be involved in preventing CDC25 from activating $\mathrm{CDC} 2 / \mathrm{CyclinB}$ and initiating mitosis [22]. CHK1 binds to CDC25C and phosphorylates CDC25C at Ser-216, which results in the 14-3-3 protein binding to CDC25C [23]. The Ser-216 site of CDC25C is the target of DNA damage repair checkpoints. In this study, we found that MUS81-deficient ovarian cancer was associated with increased sensitivity to X-ray irradiation and Olaparib treatment, and the CHK1 signaling pathway-related proteins underwent a significant changes, and CDC25 phosphorylation prevents the dephosphorylation of $\mathrm{CDC} 2$, resulting in inactivation of CDC2 kinase and preventing cell cycle entry into mitosis. An essential step in the cell cycle transition from $\mathrm{G} 2$ to mitosis includes the activation of the cell division cycle protein $2(\mathrm{Cdc} 2) /$ cyclin B complex[24].Therefore, Cdc2/cyclin B activity was inhibited and cells were arrested in G2/M phase. The cyclinB1/Cdk1 complex regulates mitotic progression by translocating to the nucleus [25, 26]. Recent work unveiled novel mechanisms that governed the localization of cyclinB1/Cdk1 and its regulator Cdc25C [27-29].

PARP inhibitors such as Olaparib are currently a novel targeted drugs for the treatment of BRCA-mutant ovarian cancer. The use of an HR repair defect mechanism leads to cell cycle arrest and apoptosis [30]. Inhibition via gene targeting inhibition in BRCA-proficient tumors in combination with Olaparib could result in a synergistic increase in DNA damage and G2/M cell-cycle checkpoint defects, which may allowed cells to enter mitosis despite the accumulation of DNA damage, ultimately causing mitotic catastrophe [31]. In previous studies, inhibition of MUS81 was observed at the cellular level. Inhibition of MUS81 significantly inhibited HR activity and increased the sensitivity of epithelial ovarian cancer to Olaparib. Furthermore, in this study, we demonstrated that MUS81-deficient ovarian cancer regulated the G2/M phase checkpoint protein CyclinB by activating the CHK1 signaling pathway, which in turns, increased the sensitivity of ovarian cancer cells to radiotherapy and Olaparib treatment. This represents a new use for Olaparib in the treatment of non-BRCA mutant ovarian cancer. By inhibiting of the expression of the MUS81 gene, the activity of $\mathrm{HR}$ is affected, thereby improving the treatment effect of Olaparib. An inhibitor of MUS81 developed by the Masaryk University Mgr. Lumír Krejčí, Ph.D research team is in the clinical trial stage, and could be used in the future to address issues with ovarian cancer treatment.

This study investigated a mechanism of targeted suppression of epithelial ovarian cancer, in which MUS81 regulated DNA damage repair and cell cycle checkpoints. In many contexts, MUS81 may be used as a novel target in EOC and may provide a new insight for the targeted-specific treatment of ovarian cancer. Further studies are warranted to elucidate the effect of MUS81-targeted inhibition combined with PARP inhibition in vivo, in vitro and in future clinical trials.

\section{Acknowledgements}

This work was supported by a grant from the National Natural Science Foundation of China (Grant No. NSF-81772808, 81572552 and 81772774).

\section{Competing Interests}

The authors have declared that no competing interest exists.

\section{References}

1. Siegel RL, Miller KD, Jemal A. Cancer statistics, 2018. CA Cancer J Clin. 2018; 68: 7-30.

2. Colombo PE, Fabbro M, Theillet C, Bibeau F, Rouanet P, Ray-Coquard I Sensitivity and resistance to treatment in the primary management of epithelial ovarian cancer. Crit Rev Oncol Hematol. 2014; 89: 207-16.

3. Haber JE, Heyer WD. The fuss about Mus81. Cell. 2001; 107: 551-4.

4. Boddy MN, Lopez-Girona A, Shanahan P, Interthal H, Heyer WD, Russell P. Damage tolerance protein Mus81 associates with the FHA1 domain of checkpoint kinase Cds1. Mol Cell Biol. 2000; 20: 8758-66.

5. Pfander B, Matos J. Control of Mus81 nuclease during the cell cycle. FEBS Lett. 2017; 591: 2048-56.

6. Hanada K, Budzowska M, Davies SL, van Drunen E, Onizawa H, Beverloo $\mathrm{HB}$, et al. The structure-specific endonuclease Mus81 contributes to replication restart by generating double-strand DNA breaks. Nat Struct Mol Biol. 2007; 14: 1096-104.

7. Jackson SP, Bartek J. The DNA-damage response in human biology and disease. Nature. 2009; 461: 1071-8.

8. Hustedt N, Durocher D. The control of DNA repair by the cell cycle. Nat Cell Biol. 2016; 19: 1-9.

9. Hiraoka D, Aono R, Hanada S, Okumura E, Kishimoto T. Two new competing pathways establish the threshold for cyclin-B-Cdk1 activation at the meiotic G2/M transition. J Cell Sci. 2016; 129: 3153-66.

10. Hsu JD, Kao SH, Ou TT, Chen YJ, Li YJ, Wang CJ. Gallic acid induces G2/M phase arrest of breast cancer cell MCF-7 through stabilization of p27(Kip1) attributed to disruption of p27(Kip1)/Skp2 complex. J Agric Food Chem. 2011; 59: 1996-2003.

11. Lindqvist A, Rodriguez-Bravo V, Medema RH. The decision to enter mitosis: feedback and redundancy in the mitotic entry network. J Cell Biol. 2009; 185: 193-202.

12. Kikuchi I, Nakayama Y, Morinaga T, Fukumoto Y, Yamaguchi N. A decrease in cyclin B1 levels leads to polyploidization in DNA damage-induced senescence. Cell Biol Int. 2010; 34: 645-53.

13. Xie S, Zheng H, Wen X, Sun J, Wang Y, Gao X, et al. MUS81 is associated with cell proliferation and cisplatin sensitivity in serous ovarian cancer. Biochem Biophys Res Commun. 2016; 476: 493-500. 
14. Zhong A, Zhang H, Xie S, Deng M, Zheng H, Wang Y, et al. Inhibition of MUS81 improves the chemical sensitivity of olaparib by regulating MCM2 in epithelial ovarian cancer. Oncol Rep. 2018; 39: 1747-56.

15. Dehe PM, Gaillard PH. Control of structure-specific endonucleases to maintain genome stability. Nat Rev Mol Cell Biol. 2017; 18: 315-30.

16. West SC, Chan YW. Genome Instability as a Consequence of Defects in the Resolution of Recombination Intermediates. Cold Spring Harb Symp Quant Biol. 2018.

17. Culligan KM, Robertson CE, Foreman J, Doerner P, Britt AB. ATR and ATM play both distinct and additive roles in response to ionizing radiation. Plant J. 2006; 48: 947-61.

18. Weimer AK, Biedermann S, Harashima H, Roodbarkelari F, Takahashi N, Foreman J, et al. The plant-specific CDKB1-CYCB1 complex mediates homologous recombination repair in Arabidopsis. EMBO J. 2016; 35: 2068-86.

19. Wu F, Su SC, Tan GQ, Yan L, Li TY, Zhang HL, et al. Mus81 knockdown sensitizes colon cancer cells to chemotherapeutic drugs by activating CHK1 pathway. Clin Res Hepatol Gastroenterol. 2017; 41: 592-601.

20. Carr AM, Moudjou M, Bentley NJ, Hagan IM. The chk1 pathway is required to prevent mitosis following cell-cycle arrest at 'start'. Curr Biol. 1995; 5: 1179-90.

21. Kumagai A, Guo Z, Emami KH, Wang SX, Dunphy WG. The Xenopus Chk1 protein kinase mediates a caffeine-sensitive pathway of checkpoint control in cell-free extracts. J Cell Biol. 1998; 142: 1559-69.

22. Jackson JR, Gilmartin A, Imburgia C, Winkler JD, Marshall LA, Roshak A. An indolocarbazole inhibitor of human checkpoint kinase (Chk1) abrogates cell cycle arrest caused by DNA damage. Cancer Res. 2000; 60: 566-72.

23. Sanchez Y, Wong C, Thoma RS, Richman R, Wu Z, Piwnica-Worms H, et al. Conservation of the Chk1 checkpoint pathway in mammals: linkage of DNA damage to Cdk regulation through Cdc25. Science. 1997; 277: 1497-501.

24. Sun WJ, Huang H, He B, Hu DH, Li PH, Yu YJ, et al. Romidepsin induces $\mathrm{G} 2 / \mathrm{M}$ phase arrest via Erk/cdc25C/cdc2/cyclinB pathway and apoptosis induction through JNK/c-Jun/caspase3 pathway in hepatocellular carcinoma cells. Biochem Pharmacol. 2017; 127: 90-100.

25. Bailly E, Pines J, Hunter T, Bornens M. Cytoplasmic accumulation of cyclin B1 in human cells: association with a detergent-resistant compartment and with the centrosome. J Cell Sci. 1992; 101 ( Pt 3): 529-45.

26. Hagting A, Jackman M, Simpson K, Pines J. Translocation of cyclin B1 to the nucleus at prophase requires a phosphorylation-dependent nuclear import signal. Curr Biol. 1999; 9: 680-9.

27. Takizawa CG, Morgan DO. Control of mitosis by changes in the subcellular location of cyclin-B1-Cdk1 and Cdc25C. Curr Opin Cell Biol. 2000; 12: 658-65.

28. Graves PR, Yu L, Schwarz JK, Gales J, Sausville EA, O'Connor PM, et al. The Chk1 protein kinase and the Cdc25C regulatory pathways are targets of the anticancer agent UCN-01. J Biol Chem. 2000; 275: 5600-5.

29. Huang $\mathrm{H}, \mathrm{Hu}$ M, Zhao R, Li P, Li M. Dihydromyricetin suppresses the proliferation of hepatocellular carcinoma cells by inducing $\mathrm{G} 2 / \mathrm{M}$ arrest through the Chk1/Chk2/Cdc25C pathway. Oncol Rep. 2013; 30: 2467-75.

30. Cortesi L, Toss A, Cucinotto I. Parp Inhibitors for the Treatment of Ovarian Cancer. Curr Cancer Drug Targets. 2018.

31. Karakashev S, Zhu H, Yokoyama Y, Zhao B, Fatkhutdinov N, Kossenkov AV, et al. BET Bromodomain Inhibition Synergizes with PARP Inhibitor in Epithelial Ovarian Cancer. Cell Rep. 2017; 21: 3398-405. 